\title{
Un magazine
}

Pour les jeunes des pays en développement

Jacqueline Kergueno

\section{CpenEdition}

\section{Journals}

Édition électronique

URL : http://journals.openedition.org/ries/4277

DOI : $10.4000 /$ ries. 4277

ISSN : 2261-4265

Éditeur

Centre international d'études pédagogiques

Édition imprimée

Date de publication : 1 juin 1994

Pagination : 95-98

ISSN : $1254-4590$

Référence électronique

Jacqueline Kergueno, « Un magazine », Revue internationale d'éducation de Sèvres [En ligne], 02 | 1994, mis en ligne le 17 avril 2015, consulté le 19 avril 2019. URL : http://journals.openedition.org/ries/4277 ; DOI : 10.4000/ries.4277

Ce document a été généré automatiquement le 19 avril 2019

(c) Tous droits réservés 


\title{
Un magazine
}

Pour les jeunes des pays en développement

\author{
Jacqueline Kergueno
}

1 On peut être lecteur débutant à sept ans mais aussi, pour diverses raisons, mal maîtriser la lecture à onze ans, à quatorze ans et plus tard. D'une certaine façon, la situation est la même : lire représente une épreuve et le découragement n'est pas loin. Il faudra du temps et des moyens pour faire découvrir à ce lecteur qu'à partir d'un certain effort, l'écrit peut être gratifiant. Il lui faudra une motivation, un projet personnel pour le décider à faire cet effort. Parmi les moyens qui l'aideront, il existe une démarche éditoriale qui aide à entrer dans l'écrit, et à y rester, sans se sentir en situation d'échec.

2 Les équipes de rédaction de Bayard Presse travaillent depuis vingt ans pour mieux comprendre le comportement du lecteur débutant inexpérimenté ou ayant peu de goût pour la lecture. Il s'en est suivi toute une réflexion pour améliorer le contact avec l'écrit. Un long et patient travail a porté essentiellement sur les repères visuels, les rythmes, le rapport texte-images, la lisibilité du texte, le confort de lecture.

\section{L'exemple de « Planète Jeunes »}

Une étude dans les pays d'Afrique francophone et de l'océan Indien nous a permis de cerner les attentes quant à l'écrit chez des jeunes qui ont des intérêts communs, des besoins dus au manque de supports écrits. Il fallait tenir compte de ces demandes mais aussi jongler avec de nombreuses contraintes. Il fallait réaliser un magazine pas trop coûteux, créant des habitudes de lecture, revenant à un rythme régulier entre les mains des lecteurs, qui tienne compte de leur peu d'expérience de l'écrit et qui réponde enfin globalement à des attentes nouvelles que nous avons identifiées. Ces lecteurs désirent comprendre ce qui se passe dans le monde, ne pas en être exclus et se reconnaître comme faisant partie d'une entité « jeunes du monde ». Ces jeunes - scolarisés - s'ils partagent la même difficulté d'apprendre à vivre, ont aussi en commun le goût de la tenue vestimentaire, aiment les mêmes musiques, les mêmes chanteurs et acteurs. Ils cherchent 
surtout des repères, des valeurs en accord avec leur perception de la vie, avec leurs projets.

Comment réaliser une publication qui intéresse les jeunes en général et chaque jeune en particulier? Un projet de magazine en couleurs mais accessible à chacun du point de vue économique? Un mensuel immédiatement gratifiant pour un lecteur inexpérimenté, offrant plusieurs niveaux de lecture et de sensibilité, pour ces jeunes de treize à dix-huit ans?

5 Pour créer un tel journal, une petite équipe internationale s'est mise au travail, composée notamment d'Africains. Elle s'est appuyée sur un large réseau de correspondants dans le monde mais aussi sur l'expérience des journalistes. Pour que ce projet soit possible, il a fallu faire un montage économique spécial: créer l'association Planète Jeunes - les partenaires étant des éditeurs de presse en Afrique, dans l'océan Indien, associés à la production et à la diffusion du magazine mais aussi des associations humanitaires, des ministères et divers parrainages.

\section{Créer une relation de confiance avec le lecteur}

6 Planète Jeunes est un mensuel de quarante-quatre pages en couleur. Imaginons un lecteur peu habitué à lire et qui découvre ce magazine. Le premier contact est sensoriel. Le regard est immédiatement attiré par la couverture. Il faut que l'image lui parle, que le titre corresponde au projet, que les titrages soient suggestifs et lui donnent envie d'ouvrir le journal. Il faut qu'il ait envie de le saisir, de le sentir entre ses mains: le format, la souplesse, la couleur, l'épaisseur et le grain du papier sont autant d'atouts.

7 Mais le lecteur doit avoir le sentiment que cet objet est aussi à sa mesure du point de vue des contenus: le sommaire lui indique clairement quels sont les thèmes qu'il va rencontrer et sous quelle forme ils sont abordés. Le rapport images-texte ne doit pas être décourageant, il faut que par le simple fait de feuilleter ce journal, le lecteur inexpérimenté se sente en confiance. Pour arriver à ce résultat, l'équipe de rédaction doit traquer, page à page, ce qui pourrait donner un sentiment d'échec. Le lecteur doit comprendre au premier coup d'œil le fonctionnement du journal, savoir où commence et où s'arrête chaque rubrique, quel est le genre de chaque rubrique: informatif ou narratif? Littéraire ou scientifique ? Humoristique ou pédagogique ? Le logo, le titrage, le traitement de l'aspect visuel des pages doivent être explicites.

Le public doit d'abord sentir qu'on s'adresse à lui et pas à un public plus jeune ou plus âgé. Pour lui donner envie de lire, il faut savoir qu'une page trop chargée de texte s'avère décourageante, de même que le manque d'espaces (blancs, alinéas, intertitres) ou le manque d'illustrations. Un intérêt particulier est accordé au choix des thèmes qui peuvent correspondre à une information dont le lecteur a besoin, à un sujet qui le préoccupe. Ce thème et ce document peuvent l'aider en classe, correspondre à une actualité qui l'intéresse (un reportage sur une vedette qu'il apprécie tout particulièrement), à un débat qui l'interpelle. Plus un lecteur lit difficilement, plus il a besoin d'être stimulé. Pour cela il faut faire appel à sa curiosité autant qu'à ses sentiments. S'il se sent touché par le ton d'un récit, s'il éprouve une émotion, il continue sa lecture, il y revient. 


\section{Quelle pédagogie de la lecture?}

9 Comment sélectionner des informations sans toute lire quand on n'aime pas lire? Entrer dans la page, c'est promener son œil dans tous les sens pour savoir si l'on va éprouver le désir d'y rester. Même si les thèmes l'intéressent, le lecteur lent sait qu'il lui faudra un certain temps avant de s'approprier des informations. Tout va tellement plus vite avec les moyens audiovisuels! Il va essayer d'obtenir des informations en lisant le moins possible.

Et si nous étions complices de ce lecteur-là ? Si nous l'aidions à trouver des attitudes de compensation pour le conduire peu à peu à lire le texte sans trop d'efforts ci surtout sans trop d'appréhension...

Proposons-lui plusieurs entrées dans la page, plusieurs niveaux de lecture. Tout d'abord l'image va retenir l'intérêt du lecteur : une image claire, lisible, informative, de qualité, en rapport étroit avec le thème et aussi séduisante que possible. Ensuite, un premier niveau de lecture facile : par le titre, le " chapeau », les intertitres, les légendes des images.

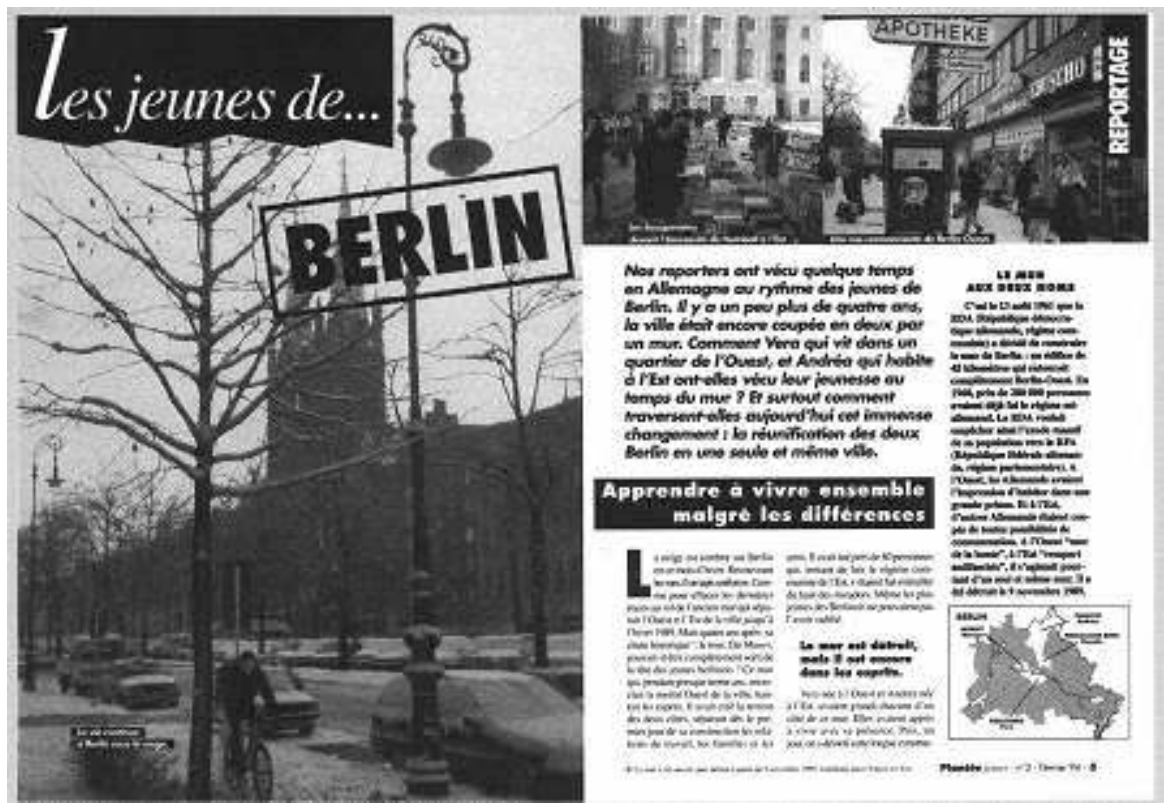

Les textes, que le lecteur soit expérimenté ou non, doivent apporter à eux seuls de premières informations. Ils doivent lui laisser l'impression qu'il a compris, qu'il a appris quelque chose. Même s'il ne lit pas toute la page, il ne quittera pas son magazine sur un sentiment d'échec. Ces premiers textes qui vont être lus doivent être assez incitatifs pour donner à un lecteur moyen l'envie d'en lire davantage.

Le texte principal n'obéit pas toujours aux mêmes règles selon le genre auquel il appartient. Ce n'est pas parce que le lecteur est malhabile que le texte doit être simpliste. Plus un lecteur lit difficilement, plus il faut chercher à lui proposer un texte intéressant. Il faut le séduire, le captiver, le retenir, faire naître en lui l'envie d'être plus performant, de maîtriser techniquement cet outil : l'écrit. Lui faire entrevoir qu'il est à la porte d'un univers qui lui promet une infinie variété de satisfactions. Exercice difficile pour l'auteur : proposer un texte simple mais captivant. Il faut au départ une vraie intuition créative, un vrai travail d'auteur pour les textes littéraires. Et des professionnels exigeants pour les textes informatifs. Alors intervient une analyse détaillée de tout ce qui nuit à la compréhension, la chasse aux phrases trop longues, incises, lourdeurs inutiles, la traque 
au mot « inconnu » s'il n'est éclairé par le contexte, aux complaisances littéraires si elles nuisent à la clarté. Dans un dialogue, on tiendra compte du fait qu'il faut identifier un locuteur avant ce qu'il dit.

14 Pour cela, il a fallu réfléchir, chercher, "creuser ", expérimenter auprès de groupes de lecteurs «tests». Trouver les cent façons de contourner la difficulté, s'appuyer sur le talent des auteurs, des illustrateurs, des photographes, maquettistes, photograveurs, imprimeurs, tous créatifs. La technique bien utilisée est un précieux outil pour faire mieux lire. La mise en page, la grosseur des caractères, le rapport des textes entre eux sont vérifiés, voire transformés au fur et à mesure que les pages se forment et cela dans le sens d'une aide à la lecture. Alors lira, lira pas? Quand le journal imprimé parviendra à ses lecteurs, il leur apportera peut-être le sentiment d'appartenir à une vaste planète, la planète des jeunes, et ils s'y sentiront peut-être un peu moins seuls. Espérons qu'elle leur donnera envie de lire de plus en plus, d'être créatifs en participant largement à l'élaboration de leur propre journal, en leur donnant le désir un jour de créer leur propre production.

\section{RÉSUMÉS}

Présentation d'une revue avec les principes pédagogiques qui la sous-tendent pour les jeunes ayant peu de contact avec l'écrit.

INDEX

Mots-clés : lecture, périodique

Index géographique : Pays en développement, Afrique

\section{AUTEUR}

JACQUELINE KERGUENO

Bayard Presse International 